\title{
Approximate Connectivity Graph Generation in Mobile Ad Hoc Radio Networks
}

\author{
Christopher L. Barrett ${ }^{1}$ \\ Madhav V. Marathe ${ }^{1}$ \\ Los Alamos National Laboratory \\ Los Alamos NM 87545 \\ Email: barrett, marathedlanl.gov
}

\author{
D. Charles Engelhart ${ }^{1,2}$ \\ Anand Sivasubramaniam \\ Penn. State University \\ University Park PA 16802 \\ Email: engelhar, anand@cse.psu.edu
}

\begin{abstract}
Simulation is a widely used technique in the design and evaluation of mobile ad-hoc networks. However, time and space constraints can often limit the extent of the size, complexity and detail of the networks that can be simulated. Approximations in the system model can possibly alleviate this problem, but we need to be careful about how much accuracy is compromised when employing them.

This paper specifically focuses on one aspect of simulation cost that is incurred in the computation of the connectivity graph that is used to describe what mobile nodes can communicate with whom. Since such a graph is recomputed frequently during the simulation, we explore alternatives to computing this graph exactly and their accuracy in capturing the actual graph properties.

We investigate three approximation alternatives to compute graph connectivity, and propose metrics for expressing their deviations from the actual graph. In addition, the graphs generated by these approximations are compared to the original by examining two previously proposed graph measures - the degree and clustering coefficient distributions. Results indicate that these approximations can be quite effective in avoiding repeated calculation of exact graph connectivity.
\end{abstract}

\section{Introduction}

Simulation plays a crucial role in the design and evaluation of networking protocols, routing mechanisms, and mobility considerations of ad hoc wireless networks. Since these networks are still in their infancy, there are numerous parameters to study. Many of which are virtually impos-

\footnotetext{
${ }^{1}$ The work is supported by the Department of Energy under Contract W-7405-ENG-36.

${ }^{2}$ Work done while the author was a graduate research student in the Basic and Applied Simulation Science Group at Los Alamos National laboratory.
}

sible to investigate on a large scale in a real deployment or to model all the complex interactions analytically without loss in accuracy. Simulators serve as convenient nonintrusive tools for studying such networks, and many such tools $[11,8]$ are in wide use today by several researchers.

A main problem with simulation is the high cost incurred in studying large mobile systems, some of which may even be impossible to model because of their high storage requirements and processing time costs. As the number of nodes in the network grows, routing protocols need to determine the evolving connectivity (who can communicate with whom) between these nodes, which can grow quadratically (we will call this graph connectivity in the rest of this paper). It has been shown [9] that the cost of simulating the ad hoc routing protocol itself does not grow as fast.

Further, the graph connectivity needs to be recomputed as nodes move leading to breaking of existing links and at the same time formation of new connections. Thus, it is important to develop better (more scalable) graph connectivity determination techniques in order to simulate much larger networks than is possible with current simulators $[11,8]$. In particular, we need to determine the set of neighbors that a node can communicate with at each instant to broadcast messages to them (which many protocols rely on).

This paper explores techniques for calculating graph connectivity between mobile nodes, which can incur lower computation costs, at a possible loss in accuracy. We also analyze how these techniques will perform on a large distributed system by investigating the workload balance on a given processing element as well as the amount of communication between processing elements.

We investigate three simple techniques that can be used for such approximation. The quality of these approximations is evaluated using graph metrics studied by previous researchers $[2,3]$ as well new metrics proposed here. We demonstrate that the techniques can quite closely track the actual graph connectivity. We also show that these approximations can be used repetitively on successive time steps in the context of ad-hoc networks, and only occasionally need 
to recalculate actual connectivity to ensure errors do not become too large.

\section{Graph Generation Methods}

We studied the graphs formed via radio network connectivity. When two nodes can communicate we say that they are connected, or there is an edge between them. We studied three synthetic graph generation methods and a reasonably realistic method.

Most current network simulators do not allow for a connectivity graph to be given as input to the simulator $[11,8]$. This limits the flexibility of such simulators. It would be preferable to be able to supply a set of possible communications to the simulator so that some outside source can remove edges that are blocked by physical terrain.

There is the risk that some of the effects of radio interference will be lost in such a scheme, but the majority of such effects can easily be modeled in the simulator at the time of communication, edges can simply be removed if connected nodes are interfering with one another.

One of our main ideologies when building a very large scale ad hoc network simulator (on the order of a million nodes) is that we cannot afford to perform every interference computation exactly. We instead would prefer a reasonable approximation of such interactions that can be computed efficiently that will still capture the dynamics of the system on a macroscopic level.

It is not sufficient to simply trust that this level of abstraction is acceptable or to claim that it is not without further evidence. A future study comparing not only how using a graph as input to a network simulator effects the macroscopic network behavior but also how that coupled with these approximate graphs will effect the network needs to be done in order to resolve these issues. To these ends we are working with the people at Qualnet to add the required functionality to their simulator.

\subsection{Exact Connectivity}

This model, which we label the exact connectivity of the graph, is the most realistic of the connectivity methods we considered. To determine whether a pair of nodes is connected we simply look at the Euclidean distance between them, which given two points $\left(x_{i}, y_{i}\right),\left(x_{j}, y_{j}\right)$, it is simply $\operatorname{sqrt}\left(x_{i}-x_{j}\right)^{2}+\left(y_{i}-y_{j}\right)^{2}$. If it is less than the radio range then they are connected, otherwise they are not. The only parameter for this method is the radio range, $R_{a}$.

This model mirrors the two-ray ground propagation model, where received power is proportional to the inverse of square of the distance between nodes up to a limiting distance and proportional to the inverse of the fourth power of the distance beyond that threshold. In the absence of inter- ference this yields a connectivity range for each node that depends on the Euclidean distance between nodes. Because this model is not a perfect representation of reality and the radio interference is not accounted for our exact model can be seen as an approximation itself. For the remainder of the paper, we will use this model as the base model for comparison.

Computationally this model requires two subtractions, two multiplications, an addition and a comparison for each possible pair of nodes, of which there are $\left(\begin{array}{l}n \\ 2\end{array}\right)$ given $n$ nodes. This is assuming all links are symmetric, which occurs if the radio ranges for each node are equal. Also there is a trivial initialization cost of one multiplication to square the radio range in order to avoid having to calculate a square root for every comparison.

All of these calculations are made at every time-step, although there are methods of computing the changes to the node distance caused by each nodes movement which simplifies things slightly.

In a large distributed system the naive approach of assigning sets of nodes to processing elements by simply dissecting the space into quadrants yields reasonable load balance if the nodes are distributed uniformly over the space, but cannot guarantee any bounds on load balance. If the quadrants are assigned such that their diameter is approximately the radio range then a given work unit will only have to communicate with the eight work units corresponding to the eight adjacent quadrants.

There is also the added computational cost of assigning each node to a work unit in this scheme. That requires two divisions for each node at each time step.

\subsection{Manhattan Distance Metric Connectivity.}

In this model we simply replace the Euclidean distance metric with the $l=1$, or Manhattan, distance metric. In this distance metric the distance between two points, $\left(x_{i}, y_{i}\right),\left(x_{j}, y_{j}\right)$, is simply the sum of the component-wise distances, $\left|x_{i}-x_{j}\right|+\left|y_{i}-y_{j}\right|$. It is worth noting that this is a legitimate distance metric. The only parameter for this method is again the radio range, but it need not be the same as the range for the actual connectivity method. We denote this range $R_{l 1}$.

The computation required is simply two subtractions, one addition and three comparisons for each pair of nodes. Similar arguments hold for workload balance and communication as in the exact method.

\subsection{Box Connectivity.}

To generate the box connectivity we split the two dimensional space the nodes are in into square sub regions of a given size, $S_{b o x}$. Let $x_{i}, y_{i}$ denote the $x$ and $y$ coordinates 
of node $i$. Then node $i$ belongs to box $(\alpha, \beta)$ iff $\left\lfloor\frac{x_{i}}{S_{b o x}}\right\rfloor=\alpha$ and $\left\lfloor\frac{y_{i}}{S_{b o x}}\right\rfloor=\beta$.

Once we have the box assignment for each node we then say that a node is connected to every other node in the same box and every node in an adjacent box. That is each node in box $(\alpha, \beta)$ is connected to every node in boxes $(\alpha \pm 1, \beta \pm 1)$. Note that some of these adjacent boxes may not actually exist for boxes on the border of the simulation.

The computation required for this method is only two divisions for each node at each time step. Also no extra computation is required to assign nodes to work units.

When a node wishes to communicate to a node outside of its work unit the message is simply passed to the eight adjacent work units without any extra computation. This again bounds the complexity of communication between workloads.

This method still has the same drawback as the two previous methods in that if the nodes are not evenly distributed in space then the amount of nodes in a work unit will not necessarily be uniform.

\section{4 k-means Cluster Connectivity.}

To calculate the clustered connectivity we first apply the $\mathrm{k}$-means clustering algorithm to our set of nodes to obtain $k$ groups of nodes. We then say that a node can communicate with any node within its cluster. We also allow a node to communicate with any node in a nearby cluster. To determine if a cluster is nearby we calculate the Euclidean distance from the centroids of each cluster. If that distance is less than some range, $R_{\text {clus }}$, then the clusters are close enough, and thus all nodes in each cluster can communicate with each other.

Initially calculating the cluster assignments is quite computationally demanding, but once the initial clusters have been assigned finding new clusters at subsequent time steps is much faster. This method will likely not be nearly as fast as the other three but is an interesting case to consider nonetheless.

Although the communication complexity is not as tightly bounded as the box method the communicating work units is still well defined. One extra benefit this method has is that the k-means algorithm will tend to balance the number of nodes in a cluster or work unit even if the nodes are not evenly distributed in the space.

\section{Graph Analysis Metrics and Measures}

There has been a large amount of study recently on the structure of real networks $[2,3]$. We use similar techniques to compare the topology of radio connectivity graphs. We also compare the graph connection matrices in both static and mobile cases.
Our strategy for comparing these graphs is two fold. First we compare the graphs exactly, that is if node $a$ is connected to node $b$ in one connectivity method but not in another we call that a dissimilarity. In this way we are, to some degree, unintentionally tying in the geographic location in the analysis process. The second type of comparison is more "statistical" in nature. Two graphs are deemed similar if they have approximately the same statistics (e.g. degree distribution).

These statistical measures are a way of capturing the macroscopic structure of the graphs and thus are good measures to test if these approximations fit well with our overall ideology of a network simulator.

\subsection{A Graph Difference Metric}

In order to compare the similarity of a pair of connectivity graphs we look at the Hamming distance between their connectivity matrices. To do this we set up a connection matrix for each graph, where a 1 is placed in row $i$ column $j$ iff node $i$ is connected to node $j$. We then convert these matrices to bit vectors in order to compare them using the familiar Hamming distance. Recall that the Hamming distance from one vector to another is the number of positions in which they differ, for example $H([1001],[0101])=2$. We can see an example of comparing the connectivity matrices of a real and approximate graph in figure 1. The Hamming distance between the two graphs shown is $H(R, A)=6$.

Hamming distance alone is not sufficient as it will vary as the number of nodes in the graphs to be compared changes. The size of the two graphs must still be the same. We want to allow for a comparison between a measure made between a pair of graphs of one size and another measure made between another pair of graphs of another size.

For example consider two pairs of graphs, the first pair has 2 nodes and the second pair has 5 nodes. In the first pair to be considered, one graph has the two nodes connected, the other does not, the Hamming distance between their connectivity matrices is 2 (because the entries for node 1 being connected to node 2 appears as well as an entry for node 2's connection to node 1 ). In the second pair of graphs imagine that all of the connections are the same except for 2 connections, so we would find the Hamming distance to be 4.

In the second pair the graphs are almost exactly the same, with only 2 differences, and the first pair are totally different. The Hamming distance alone would tell us that the second pair are more dissimilar than the first.

In order to have a metric that is invariant w.r.t. the size of the pair of graphs we simply divide by the total number of possible connections, if $n$ is the number of nodes in the graph then this denominator will be $n^{2}$. Thus we have a metric for the percentage difference between two graphs of the same size. We call this metric the percent Hamming distance and denote it $\% H$. 
Now when we look at the previous example we see that the $\% H$ of the first pair of graphs is $\frac{2}{4}=.5$ and the $\% H$ for the second set of graphs is $\frac{4}{25}=.16$. This fits much better with our intuition that the first is much more dissimilar than the second.

\subsection{A Parameterized Graph Difference Measure}

In general when comparing two graphs we will be comparing an approximate graph to a "real" one. Recall that we specify that the graph created using Euclidean distance to connect the nodes is the graph that we will be using as our real graph. In this case the differences between two graphs can be split into one of two categories. The first case is when an edge is present in the real graph and not in the approximate one, this we call a miss. Conversely an edge can be present in an approximate graph but not actually exist in the real graph, we call this case a false positive.

The percent Hamming distance treats both of these cases the same. In certain instances, it might be more important to avoid one kind of error than the other. In order to capture this, we simply add a weighting to each of the false positive and the miss counts. We then denote the weighted percent Hamming distance $\% H_{w_{f p}, w_{m}}(r, a)$ where $w_{f p}$ is the weight of a false positive, $w_{m}$ is the weight of a miss, $r$ is the real vector, and $a$ is the approximate vector. Note when $w_{f p} \neq w_{m}$ this is no longer a distance metric as it is not symmetric, that is $\% H_{w_{f p}, w_{m}}(r, a)$ is not necessarily equal to $\% H_{w_{f p}, w_{m}}(a, r)$. Also note that the values for the weighted percent Hamming distance no longer range from 0 to 1 but from 0 to $\max \left(w_{f p}, w_{m}\right)$.

In simulations of mobile ad hoc networks, we expect that a false positive would have a greater negative impact on the accuracy of the simulation than a miss would. We say this because a false positive allows two nodes that cannot communicate directly to do so, whereas a miss only makes those two nodes find an alternate path in order to communicate, which is still a viable option in reality. An example calculation for this weighted percent hamming distance with $w_{f p}=3$ and $w_{m}=1$ is illustrated in Figure 1.

\section{Graph Analysis Methodology}

In this section we discuss methods of comparing graphs that are not as sensitive to geographic location. We also discuss ways of comparing our methods once mobility is introduced into the simulation. Since each of our connectivity methods is parameterized we also try to find optimal values for the parameters, which will be used for subsequent analysis of these methods.

1. Degree Distribution. The degree distribution of a graph has recently received widespread attention (see [5] for a very informative survey on this topic). We let the degree

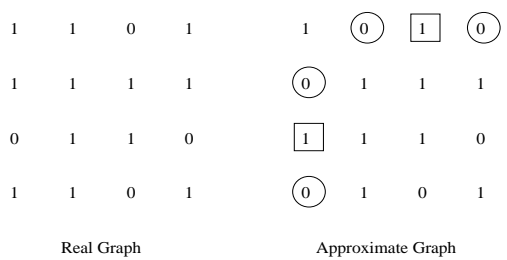

Figure 1. An illustration of the percent Hamming distance of two graphs as well as the weighted percent Hamming distance. $\% H(R, A)=6 / 16$. In the approximate graph the misses are circled and the false positives are boxed. $\% H_{3,1}(R, A)=(3 \cdot 2+4 \cdot 1) / 16$.

of a node be $d$ and the number of nodes in a graph with degree $d$ be defined as $|d|$. The degree distribution is simply a plot of $d$ versus $|d|$.

Work has been done to classify graphs using the degree distribution. Recently, a large amount of work has focused on scale free networks [2,3], or graphs with a degree distribution that follows $|d| \sim d^{-\gamma}$. The random graph model of Erdős and Rényi [7] has been studied in great detail and is known to have a degree distribution that follows a Poisson distribution.

2. Clustering Coefficient. Another widely studied graph measure is the clustering coefficient [10]. The clustering coefficient of node $i$ is defined as the number of connections between its neighbors, $n_{i}$, divided by the total possible number of connections between them, which if node $i$ has $d_{i}$ neighbors is $\left(\begin{array}{c}d_{i} \\ 2\end{array}\right)$. We consider the relationship between the degree of a node, $d_{i}$, and its clustering coefficient, $C_{i}=2 n_{i} / d_{i}\left(d_{i}-1\right)$.

It was observed in [6] that in many scale-free graphs $C(d) \sim d^{-1}$. In [4] this is instead demonstrated to be a property of hierarchical graphs.

\subsection{Parameter Tuning}

The parameter values for the approximate algorithms are not immediately obvious. To find parameter values that would make the approximate graphs as close to the base graph, we ran a series of experiments seeking to minimize the graph difference.

We used the graph difference metric mentioned above, $\% H$. We then found minimum and maximum values for the parameters for each algorithm and ran experiments with the parameter values ranging uniformly from the minimum to the maximum parameter value. We then plotted these results to obtain a parameter value to be used for the remaining experiments.

The minimum and maximum values were chosen such that at one extreme there would be no false positives and 
at the other there would be no misses. This should account for all of the reasonable approximate graphs. This was done exactly for the $l=1$ distance metric and box connectivity models, but expected values were used to find minimum and maximum values that should include a very small number of false positives or misses at the two extremes.

All of the parameter tuning experiments were run on a 1 kilometer by 1 kilometer flat square region, and 1000 nodes were simulated. The real radio range, $R_{a}$, was chosen to be 250 meters.

The $\mathrm{x}$-axis on the following graphs is the parameter we are tuning, such as $R_{l 1}$, and the y axis is the percent hamming distance between that graph and the exact one. In the case of the k-means clustering method there are two parameters to be tuned and thus the two horizontal axes are the parameters to be tuned and the vertical axis is again the percent hamming distance.

\subsubsection{Results for $R_{l 1}$ Connectivity}

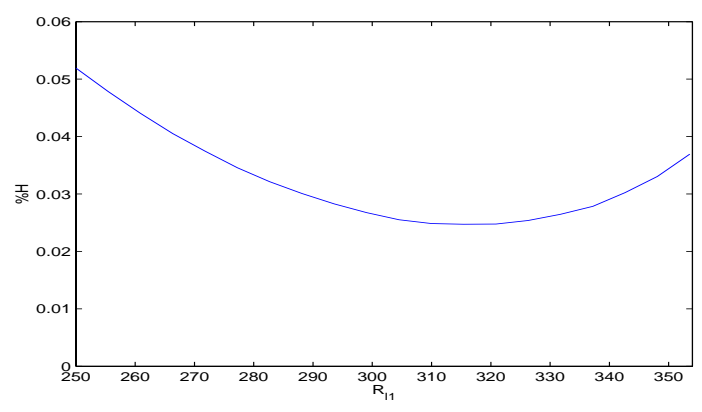

Figure 2. The parameter tuning results for the $l=1$ distance metric connectivity method.

It is easily verified that with a $R_{l 1} \leq R_{a}$ no false positives will be present in the $l=1$ distance metric method. Similarly if $R_{l 1} \geq \frac{2}{\sqrt{2}} R_{a}$ that no misses will occur. Using these bounds we see that we should let $R_{a} \leq R_{l 1} \leq \frac{2}{\sqrt{2}} R_{a}$.

The results are given in Figure 2 . We found that $R_{l 1} \approx$ $1.234 * R_{a}$ gave the minimum percent graph difference (percent hamming distance). We then proceeded to use this value for the remaining experiments.

\subsubsection{Results for Box Connectivity}

It is also clear that if $S_{b o x} \leq \frac{R_{a}}{2 \sqrt{2}}$ then no false positives will be included. Similarly if $S_{b o x} \geq R_{a}$ no misses will occur. Using these results we set $\frac{R_{a}}{2 \sqrt{2}} \leq S_{b o x} \leq R_{a}$.

The results for the box method tuning are given in figure 3 . We found that a value of $S_{b o x} \approx 0.5577 * R_{a}$ minimized the percent graph difference and thus was used for the remaining experiments.

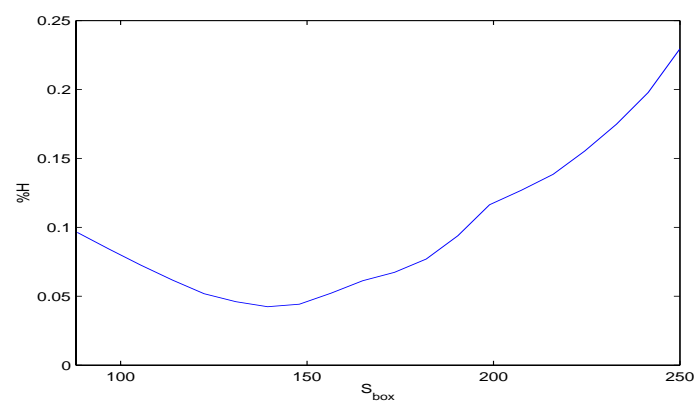

Figure 3. The parameter tuning results for the box connectivity method.

It is worth noting that these two previous bounds are tight. That is, if the minimum values for $R_{l 1}$ or $S_{b o x}$ are set any higher then false positives may occur, and similarly for the maximum values.

\subsubsection{Results for $k$-means Clustering Connectivity}

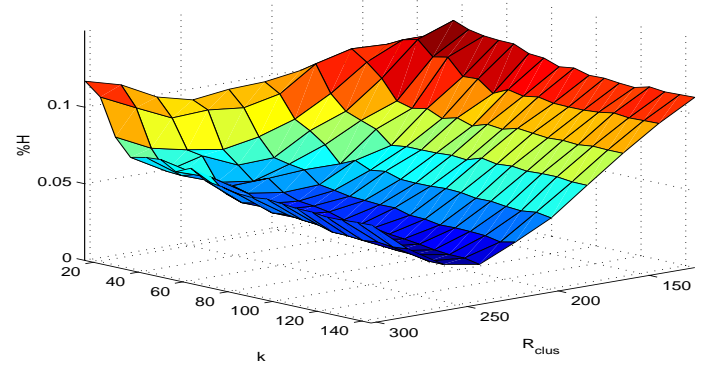

\section{Figure 4. The parameter tuning results for the clustered connectivity method.}

Because of the nature of the $k$-means clustering algorithm such tight bounds were not found for the clustered connectivity method.

We based the bounds for the clustered method on the assumption that each cluster would have the average number of nodes per cluster and thus would cover on average a set percentage of the region.

It is also worth noting that as the number of clusters, $k$, approaches the number of nodes in the system this method breaks down into the actual connectivity model if $R_{\text {clus }}=$ $R_{a}$. Because of this, instead of finding a value of $k$ to minimize the percent graph difference we looked for a percolation point. That is, we looked for an inflection in the graph where increasing the number of clusters starts producing diminishing returns.

We initially found bounds for $R_{\text {clus }}$ and $k$ to be $R_{a} / 2 \leq$ 
$R_{\text {clus }} \leq R_{a}$ and $2 *\left(\frac{s}{R_{a}}\right)^{2} \leq k \leq N$, where $s$ is the size of the region, in this case $1 \mathrm{~km}$, and $N$ is the number of nodes simulated, in this case $N=1000$. After inspecting the results we noted that the region we were concerned with was more in the region where $R_{a} / 2 \leq R_{\text {clus }} \leq 1.25 * R_{a}$ and $\left(\frac{s}{R_{a}}\right)^{2} \leq k \leq 9 *\left(\frac{s}{R_{a}}\right)^{2}$.

The results are given in figure 4. From that graph we found that the percolation point for the number of clusters occurred before $k=4 *\left(\frac{s}{R_{a}}\right)^{2}$ and the optimal value for the range was $R_{\text {clus }}=R_{a}$.

\subsection{Mobility Adaptation}

In a mobile ad hoc network simulator it would be desirable from a computational standpoint to not generate a new graph at every time interval. Instead, we would like to generate the graph every "once in a while". We call the time interval that the simulator runs on the "fine grain" time interval. We then call this graph update interval the "coarse grain" interval.

Although it is not necessary for a simulator to run in such a discrete manner we note that many, if not most, very large scale simulators run on such discrete time intervals.

Since we would like to not have to update the graph during every "fine grain" interval we would like to study the consequences of such an action. To do this we chose a fine grain interval on which to run the real connectivity model. We then ran all of the mobility models (even the real one) on a coarse grain interval and compared the graphs to the real one at every time step.

In general we would expect to see a saw tooth pattern in a graph of $\% H$ vs. time. Specifically, when the connectivity models are updated they should be very close to the actual connectivity, but as the real graph evolves over time we would expect that they would become less and less accurate until the beginning of the next coarse grain time interval. We also might expect that the peaks of each interval might not be entirely "sharp". That is we would expect that at some point the difference between the coarse representation and the actual graph will level out at some asymptotic value. At the very least the graph difference should not go above .5 or it would be better to simply use a random graph.

\section{Experimental Results}

In all of our experiments we used the same average node density, 1 node per 1000 square meters. We distributed the nodes uniformly at random over a flat square region. The real radio range, $R_{a}$, was chosen to be 250 meters.

It is worth noting that increasing the node density has the same effect as running the simulation many times, as long as the density is set fairly high there was an extremely small amount of variation between runs. Thus all of the results presented are only for a single run, but other runs were done to insure this consistency.

\subsection{Degree Distribution}

The degree distribution experiments were run on a 2 kilometer by 2 kilometer flat square region, thus 4000 nodes were simulated in order to maintain the previously stated node density. The number of nodes was increased in order to attain a smoother plot of degree distribution.

The degree distribution results are given in Figure 5. It is clear that the degree distribution is not similar to that of a scale free network.

We might expect that these degree distributions would follow the simple Poisson distribution that is exhibited by random graphs. Looking at the plot for the real connectivity model we can see that there are in fact two separate components to the degree distribution. The dominant component appears to follow a Poisson distribution with mean degree of approximately 200. The minor component is not as easily identifiable.

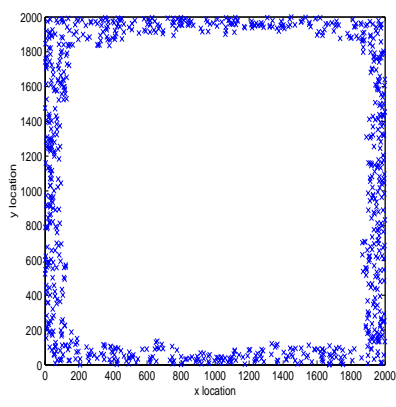

\section{Figure 6. The locations of the set of nodes with node degree less than 150 in the real connectivity model .}

To account for the appearance of the minor component we examined the set of points that were clearly outside the Poisson distribution. To do this we simply set a threshold which if a point was below we said that it was not likely part of the Poisson distribution.

The threshold value we chose was node degree 150 , so any node that has degree less than or equal to 150 we examined separately to determine if there was in fact some relationship. After plotting the spatial location of these nodes it was immediately obvious what their relationship was. It is actually fairly intuitive that the nodes on the edge of the region would have a lower degree than the nodes in the center.

The results are shown in Figure 6. It is very clear from the figure that the nodes that account for the minor component in the degree distribution are all on the periphery of the simulated region. It is worth noting that this boundary con- 


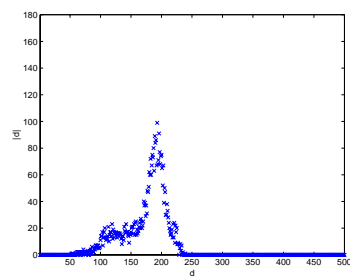

(a) actual

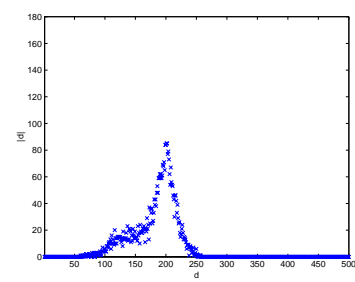

(b) $l=1$

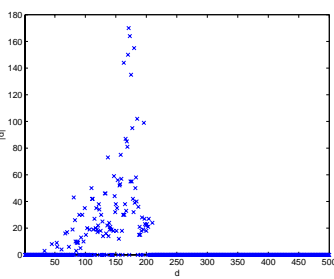

(c) box

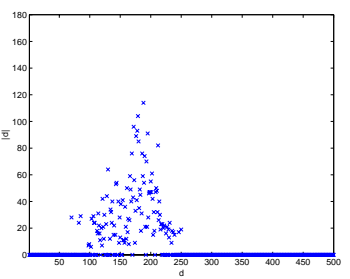

(d) clustered

Figure 5. The degree distributions for each connectivity method.

dition is a somewhat synthetic condition created in a simulation and not necessarily the case in a real radio network.

We conjecture that this minor component is either a Poisson distribution or the sum of several Poisson distributions. If this were the case the degree distribution would be the sum of two or more Poisson distributions. Then if one wanted to generate graphs similar to these graphs, one could do so by generation of a random graph with different edge probabilities for different groups of nodes in the graph to recreate this sum of Poisson distributions.

Comparing the graphs it is quite clear that the real and $l=1$ models have much smoother curves than the other two models. We attribute this to the fact that they are actually computing a distance between any two points, thus there is a nice, almost continuous, distribution of degrees.

The other two models "group" nodes and thus many nodes will have exactly the same degree and some degrees in between these "popular" degrees have very few nodes. This popularity of certain degrees can be seen by the fact that the actual $|d|$ numbers are much higher for the peaks of these two models. However, since the peaks are located in the same place it must be the case that there are some degrees with significantly less nodes belonging to them.

\subsection{Clustering Coefficient}

The clustering coefficient experiments were run on the same 2 kilometer by 2 kilometer flat square region as the degree distribution experiments, thus 4000 nodes were again simulated.

The results are given in figure 7 . The results show, as the degree distribution results did, that these graphs do not appear to exhibit the same structure that we would expect from either a scale-free or hierarchical graph.

\subsection{Mobility Adaptation}

The mobility experiments were run on a 1 kilometer by 1 kilometer area. The random way point mobility model was used with a maximum speed of 5 meters/second and a pause time of zero seconds. Each experiment was run for 10 coarse grain time intervals. The fine grain time interval was 1 second.

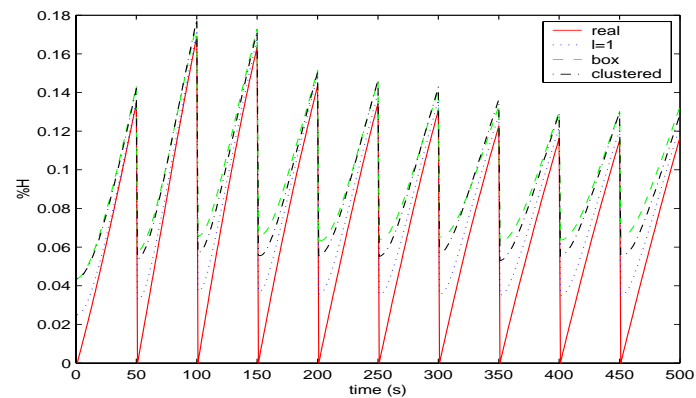

\section{Figure 8. The mobility results using a short (50 seconds) coarse grain time interval.}

The results are given for a simulation using a relatively short coarse grain time interval, 50 seconds, in figure 8 . The saw tooth pattern shows up as we expected. It is worth noting that with this time interval the Euclidean distance metric connectivity seems to always beat out the other connectivity methods. Note that the percent graph difference for the other connectivity models does not drop to zero at the coarse time steps as they are approximate models.

One interesting result is that immediately after the coarse grain update is complete the other connectivity models slowly increase their distance from the actual graph, whereas the real model has a sharp rise. Upon inspecting this we might suspect that at some point that the other models could perform better than the actual connectivity model.

To see if that was actually the case we ran an experiment with a long coarse grain update interval, 250 seconds. The results for this experiment are shown in figure 9.

With this longer interval the first point worth noting is that the percent hamming distance is quite high, sometimes almost 35\%. Even though the graphs are substantially dif- 


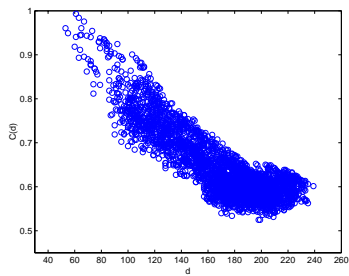

(a) actual

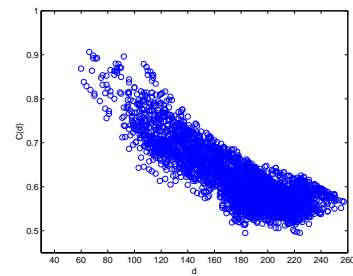

(b) $l=1$

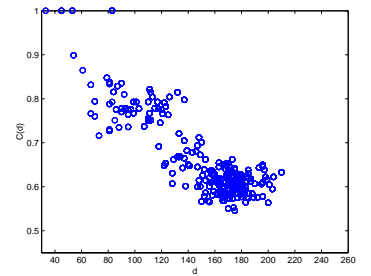

(c) box

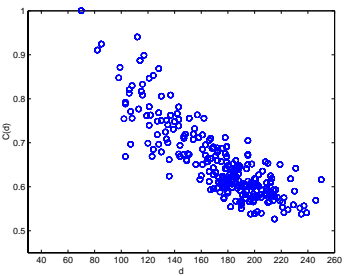

(d) clustered

Figure 7. The Clustering coefficients for each connectivity method.

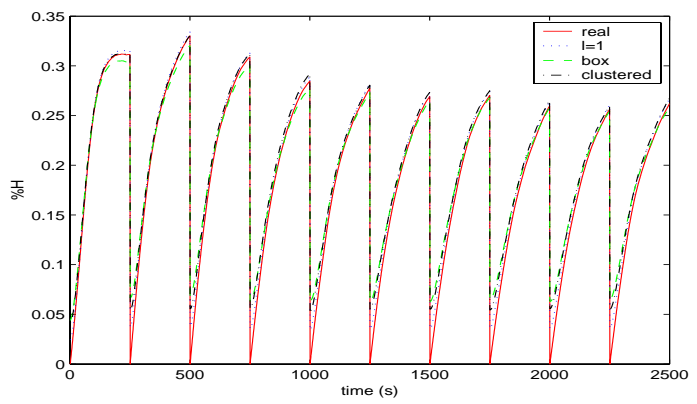

Figure 9. The mobility results using a long (250 seconds) coarse grain time interval.

ferent after this long interval it is worth noting that sometimes the other connectivity models are more accurate than the real connectivity model.

\section{Summary and Concluding Remarks}

In conclusion we find that the methods studied each have their own advantages and disadvantages.

We found that the $l=1$ distance method was the most accurate of the methods, but it does not simplify the calculations required to compute the graph. Additionally it does not reduce the space required to store the graph.

The box method can be very simple to implement, as little as a simple shift to assign boxes and a comparison to determine connections. It also has the advantage that the neighbors of each node need not be stored, instead only the box assignment needs to be stored. However, the box method is not as accurate as the $l=1$ or clustered methods.

The clustered method is quite accurate and it also reduces the amount of space required to store the graph, but initially it is quite computationally intense to compute. After computing the first graph it is easier to compute the cluster assignments but it is not nearly as simple as the other methods.
The clustered method also suffers in that the distances between cluster centers must be computed as well.

Future studies will investigate how the structural differences and similarities between these graphs relate to ad hoc network performance in a simulator such as ns or Qualnet.

\section{References}

[1] R. Albert, A.-L. Barabási. Statistical Mechanics of Complex Networks. Reviews of Modern Physics, 2001. (citeseer.nj.nec.com/4 $42178 . h t m l$ )

[2] A.-L. BARABÁSi AND R. Albert, 1999, Science 286, 509.

[3] A.-L. Barabási, R. Albert and H. Jeong, 1999, Physica A 272, 173.

[4] A.-L. BARABÁSI AND E. RAVASZ, Heirarchical organization in complex networks, preprint May 30 2002.

[5] B. Bollob Ás, 1981, Discrete Math. 33, 1.

[6] S. N. Dorogovtsev, A. V. Goltsev And J. F. F. MENDES, 2001, Los Alamos Archive condmat/0112143.

[7] P. Erdős And A. RÉnyi, 1959, Publ. Math. Debrecen 6, 290.

[8] L. Bajaj, M. Takai, R. Ahuja, K. Tang, R. Bagrodia, and M. Gerla. GloMoSim: A Scalable Network Simulation Environment. UCLA Computer Science Department Technical Report 990027, May 1999.

[9] C. Santiváñez, B. MCDonnald, I. STAVRAKAKIS, AND R. RAMANATHAN On the Scalability of Ad Hoc Routing Protocols, in Proceedings of IEEE INFOCOM'02, New York, June, 2002.

[10] D. J. Watts And S. H. Strogatz, 1998, Nature 393, 440.

[11] $n s-2$ Network Simulator. www.isi.edu/nsnam/ns, 2000 (version 2.1b8a) 